\title{
Labyrinthine Artery Aneurysm as an Internal Auditory Canal Mass
}

\author{
Rodney C. Diaz ${ }^{1}$ Thomas Konia ${ }^{2}$ James Brunberg ${ }^{3}$ \\ 1 Department of Otolaryngology-Head and Neck Surgery, University of \\ California Davis Medical Center, Sacramento, California, \\ United States \\ 2 Department of Pathology, University of California Davis Medical \\ Center, Sacramento, California, United States \\ ${ }^{3}$ Department of Radiology, University of California Davis Medical \\ Center, Sacramento, California, United States
}

\begin{abstract}
Address for correspondence Rodney C. Diaz, MD, Associate Professor, Department of Otolaryngology-Head and Neck Surgery, University of California Davis Medical Center, 2521 Stockton Boulevard, Suite 7200, Sacramento, CA 95817, United States (e-mail: rcdiaz@ucdavis.edu).
\end{abstract}

J Neurol Surg Rep 2014;75:e38-e41.

\author{
Abstract \\ Keywords \\ - labyrinthine artery \\ - internal auditory \\ artery \\ - aneurysm \\ - internal auditory \\ canal \\ - facial paralysis \\ - acoustic neuroma \\ - vestibular \\ schwannoma \\ - mass
}

We present the first case report of a labyrinthine artery aneurysm masquerading as an internal auditory canal tumor. A 72-year-old woman presented with sudden onset right facial paralysis, facial pain, hearing loss, and vertigo. She demonstrated dense rightsided facial paralysis involving all branches of the facial nerve, left beating horizontal nystagmus, and anacusis of the right ear. Magnetic resonance imaging with contrast demonstrated a $6 \times 7 \mathrm{~mm}$ peripherally enhancing lesion with lack of central uptake filling the right internal auditory canal. The patient elected to proceed with translabyrinthine approach to the internal auditory canal for definitive resection of the mass as well as to decompress the neural structures of the internal auditory canal in an attempt to recover neural function, particularly of the facial nerve. Intraoperatively, the internal auditory canal mass was resected with minimal difficulty, with intraoperative dissection notable for brisk bleeding at the medial base of the tumor just as the tumor was dissected off its medial fibrous attachments. Final pathology of the resected mass revealed a blood vessel with mucinous degeneration of the medial layer of the vessel wall, with immunohistochemical staining confirming the presence and structure of aneurysmal blood vessel.

\section{Presentation}

We present the first case report of a labyrinthine artery aneurysm masquerading as an internal auditory canal tumor. A 72-year-old woman presented to the clinic with a 1-week history of sudden onset right facial paralysis, right facial pain, hearing loss, and vertigo. On examination, she was found to have a dense right-sided facial paralysis involving all branches of the facial nerve, right facial hypoesthesia involving all branches of the trigeminal nerve, no response to whispered voice in the right ear, and left beating horizontal nystagmus. The Weber tuning fork test demonstrated sound lateralization to the left, and the Rinne tuning fork test demonstrated no ability to discern sound over the right ear and right mastoid. Behavioral audiometry revealed a complete anacusis of the right ear.

\section{Radiographic Findings}

Magnetic resonance imaging of the brain demonstrated mild expansion of the right internal auditory canal (IAC) by a mass of homogeneous signal intensity, slightly hyperintense relative to cortex on T2 images. The mass, measuring $6 \mathrm{~mm} \times 7$ $\mathrm{mm}$, filled the IAC but did not extend beyond the porus received

June 21, 2013

accepted

September 3, 2013

published online

December 12, 2013
DOI http://dx.doi.org/

10.1055/s-0033-1358796. ISSN 2193-6358. (c) 2014 Georg Thieme Verlag KG
Stuttgart · New York

License terms

$\Theta(1) \Theta \Theta$ 


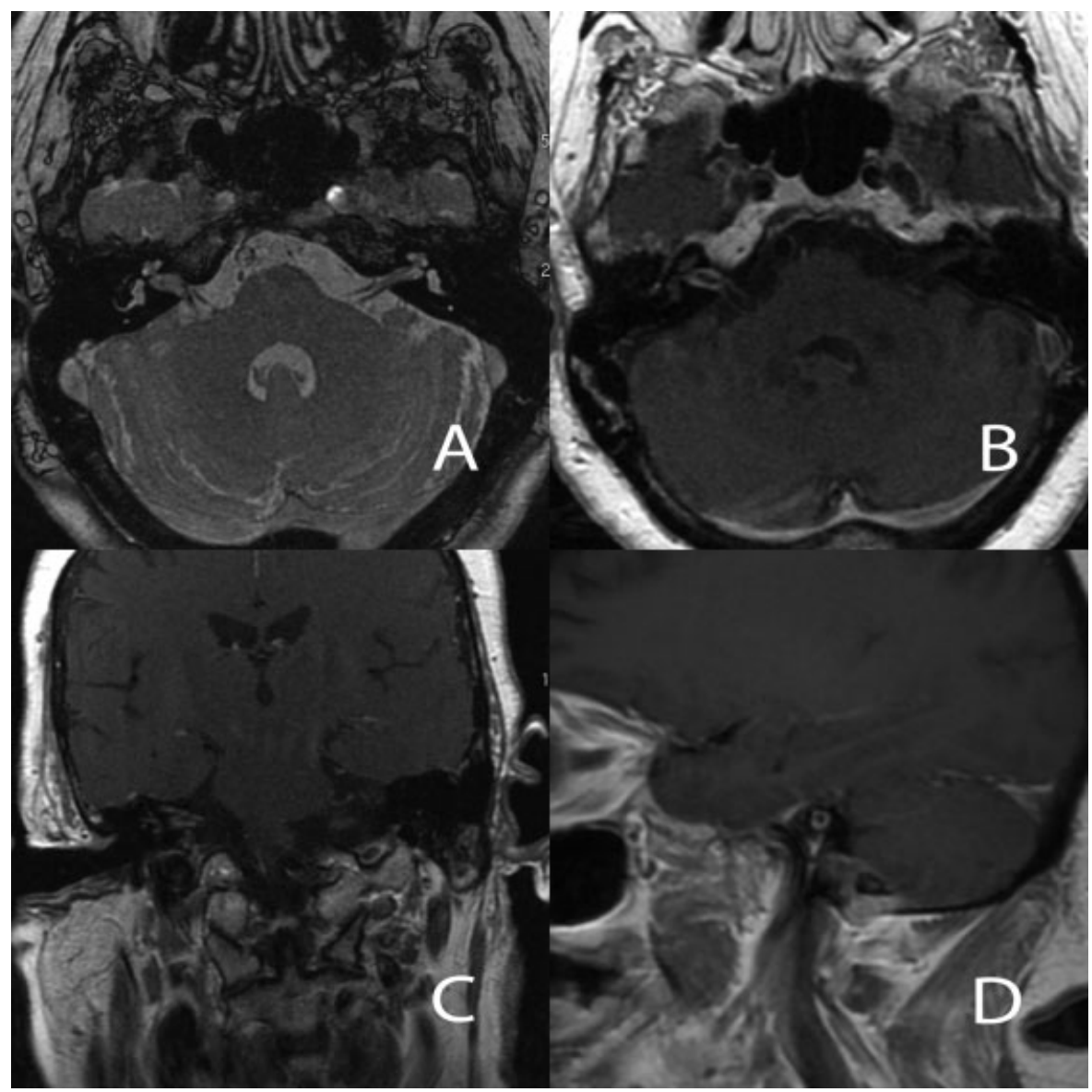

Fig. 1 (A) Magnetic resonance imaging (MRI) three-dimensional coherent oscillatory state acquisition for manipulation of imaging contrast (3D COSMIC), axial, mid-internal auditory canal (IAC), demonstrating homogeneous appearance of mass filling the IAC, with T2-weighted signal relatively isointense to that of the cerebral cortex. (B) MRI T1 with gadolinium contrast, axial, mid-IAC, demonstrating peripherally enhancing mass filling and widening the right IAC. (C) MRI T1 with gadolinium contrast, coronal, mid-IAC, demonstrating peripherally enhancing mass within the right IAC. (D) MRI T1 with gadolinium contrast, sagittal, mid-IAC, demonstrating peripherally enhancing mass within the right IAC with a distinctive "ring"-type structure verifying circumferential enhancement.

acusticus. The mass demonstrated peripheral enhancement following contrast administration (-Fig. 1). Given its location, imaging characteristics, and gadolinium enhancement, coupled with the patient's clinical presentation, the mass was thought to most likely represent a vestibular schwannoma. Peripheral enhancement with central nonenhancement also suggested a perineural inflammatory or infectious process, or less likely a metastatic or malignant process with central cyst or necrosis.

\section{Treatment Options}

All treatment options were discussed in complete detail with the patient and her husband. These included (1) observation and serial imaging of the IAC mass with primary medical treatment of facial paralysis; (2) fractionated or hypofractionated radiotherapy treatment of the IAC mass; (3) stereotactic radiotherapy treatment of the IAC mass; (4) middle fossa approach for primary surgical decompression of the IAC; and (5) surgical resection of the IAC mass for primary treatment of the mass, histopathologic diagnosis, and decompression of neural structures within the IAC. The surgical approaches were further characterized as middle fossa, retrosigmoid, and translabyrinthine approaches, with the usual advantages and admonitions specific to each approach described in detail.

After exhaustive consideration of all treatment options, and given the acute onset of symptoms, acute and complete paralysis of facial function, imaging characteristics of the IAC mass with potential to represent a malignant or metastatic process, and lack of histopathologic diagnosis of the IAC mass, the patient elected to proceed with resection of the IAC mass via a translabyrinthine approach. This approach would provide complete visualization of the IAC from fundus to porus, allow resection of mass and decompression of the IAC with no displacement of the facial nerve, and allow maximal opportunity for recovery of neural function of the facial nerve.

\section{Intraoperative Findings}

The translabyrinthine approach to the internal auditory canal was accomplished without difficulty. The IAC dura was incised and IAC contents exposed in the usual fashion, with the gross appearance of a solid nonpulsatile mass filling the IAC volume and compressing the facial nerve anterosuperiorly (-Fig. 2 ). The IAC tumor was dissected off of the effaced facial nerve with minimal difficulty. Intraoperative dissection was notable for 


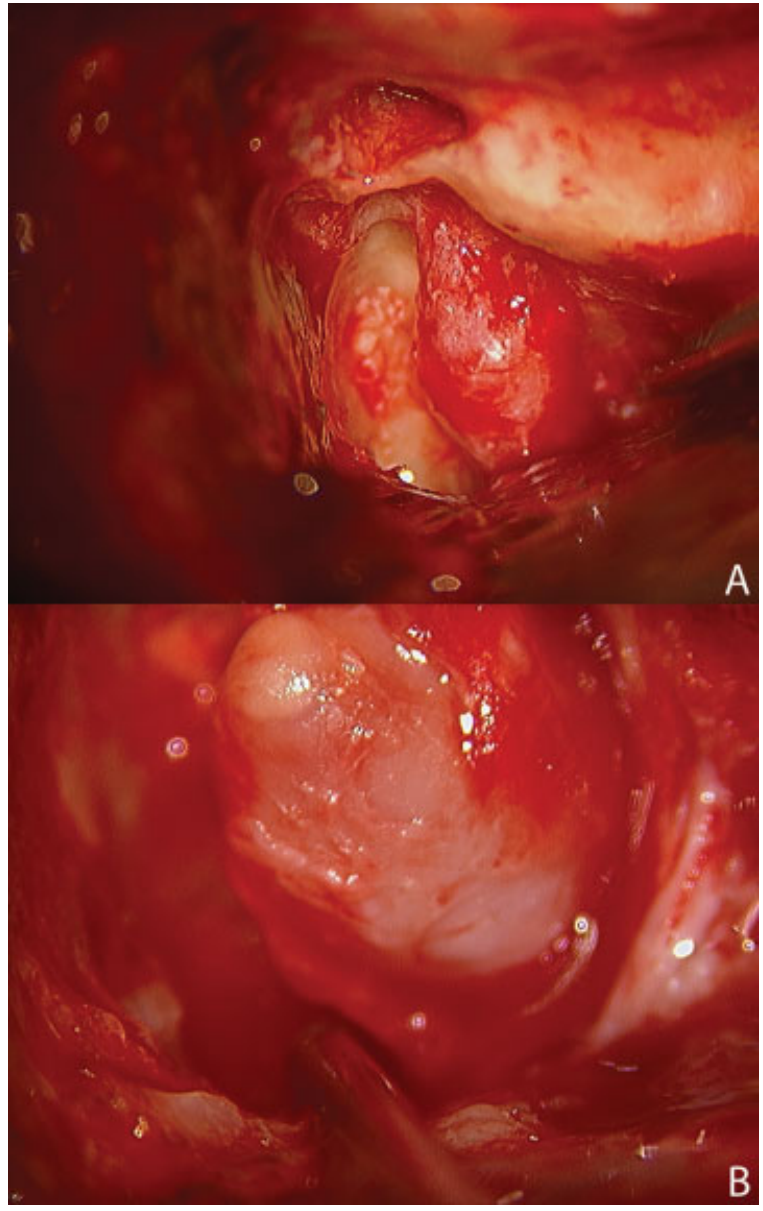

Fig. 2 (A) Intraoperative surgical view, internal auditory canal (IAC), prior to opening of the IAC dura, demonstrating mass filling the IAC. (B) Intraoperative surgical view, IAC, with tumor partially dissected, demonstrating exposed fundal end which appears solid, not vascular.

brisk bleeding at the medial base of the tumor just as the tumor was dissected off its medial fibrous attachments. A posteriori note was made of the lack of bleeding during dissection of the lateral end of the tumor out of the fundus. The facial nerve was maintained intact anatomically but never demonstrated electrical function, either at the brainstem or at the meatal foramen, either before or after dissection.

\section{Histopathology}

Final histopathology of the resected mass demonstrated a blood vessel with mucinous degeneration of the medial layer of the vessel wall. Immunohistochemical staining demonstrated positive staining to human muscle actin throughout the vessel wall, confirming the presence of smooth muscle cells within the wall of the mass, and negative staining to S-100, militating against neoplasm of neural or nerve sheath origin as the composition of the tumor specimen (-Fig. $\mathbf{3}$ ).

\section{Long-Term Results}

Despite the lack of electrical stimulability to the facial nerve before, during, and at the conclusion of intracanalicular dissection, the patient ultimately had significant success as a result of her intervention. Intraoperatively, the patient underwent gold weight implantation of the ipsilateral upper eyelid at the conclusion of her tumor dissection. She maintained a dense facial paralysis for approximately 1 year from the date of her surgery. At $\sim 15$ months, she began exhibiting minimal movement to the upper branches of the facial nerve, which progressed further over the ensuing 6 to 9 months. At $\sim 2$ years, her facial function recovery had progressed significantly to a House-Brackmann grade $3 / 6$, and her upper eyelid gold weight was explanted. At 3 years postoperatively, she has maintained a House-Brackmann grade $3 / 6$ facial function, with persistent mild to moderate synkinesis but excellent complete volitional eye closure and symmetrical upper and lower branch volitional motor function and blink, and she is pleased with her result.

The patient's initial presentation of unilateral facial pain and hypoesthesia were puzzling, given the limited extension of the enhancing mass on preoperative imaging. We did not have a straightforward explanation for these trigeminal symptoms, but the out-of-the-ordinary presentation added additional motivation to obtain definitive histopathologic diagnosis of this mass. Postoperatively, the patient maintained facial pain and hypoesthesia acutely. Her facial pain resolved rapidly and had completely resolved within 3 months. Her hypoesthesia resolved much more slowly but did resolve completely by her 2-year postoperative anniversary. As her facial pain and hypoesthesia appear to parallel the timeline of recovery of her facial function, our most compelling explanation of these symptoms is of the patient subjectively interpreting her acute facial paralysis not only as loss of motor function but also as a loss of facial sensation.

\section{Discussion}

The labyrinthine artery, also called the internal auditory artery, arises as a branch of the anterior inferior cerebellar artery, in its loop section within the cerebellopontine angle. The labyrinthine artery travels the length of the IAC and supplies the entirety of the otic capsule via its anterior vestibular, vestibulocochlear, and cochlear artery branches. ${ }^{1,2}$ Because the labyrinthine artery is the only named artery that traverses the IAC, it must by default be the arterial source of the aneurysmal mass described here.

\section{Summary}

This case represents the first reported presentation of a labyrinthine artery aneurysm within the IAC masquerading as either a schwannoma, inflammatory process, or metastatic process involving the intracanalicular vestibulocochlear or facial nerve. Recognition of the existence of the diagnostic entity of a labyrinthine artery aneurysm will allow surgeons and radiologists to include and consider such aneurysms of the labyrinthine artery within the differential diagnosis of similar clinicopathologic presentations, and it allows 


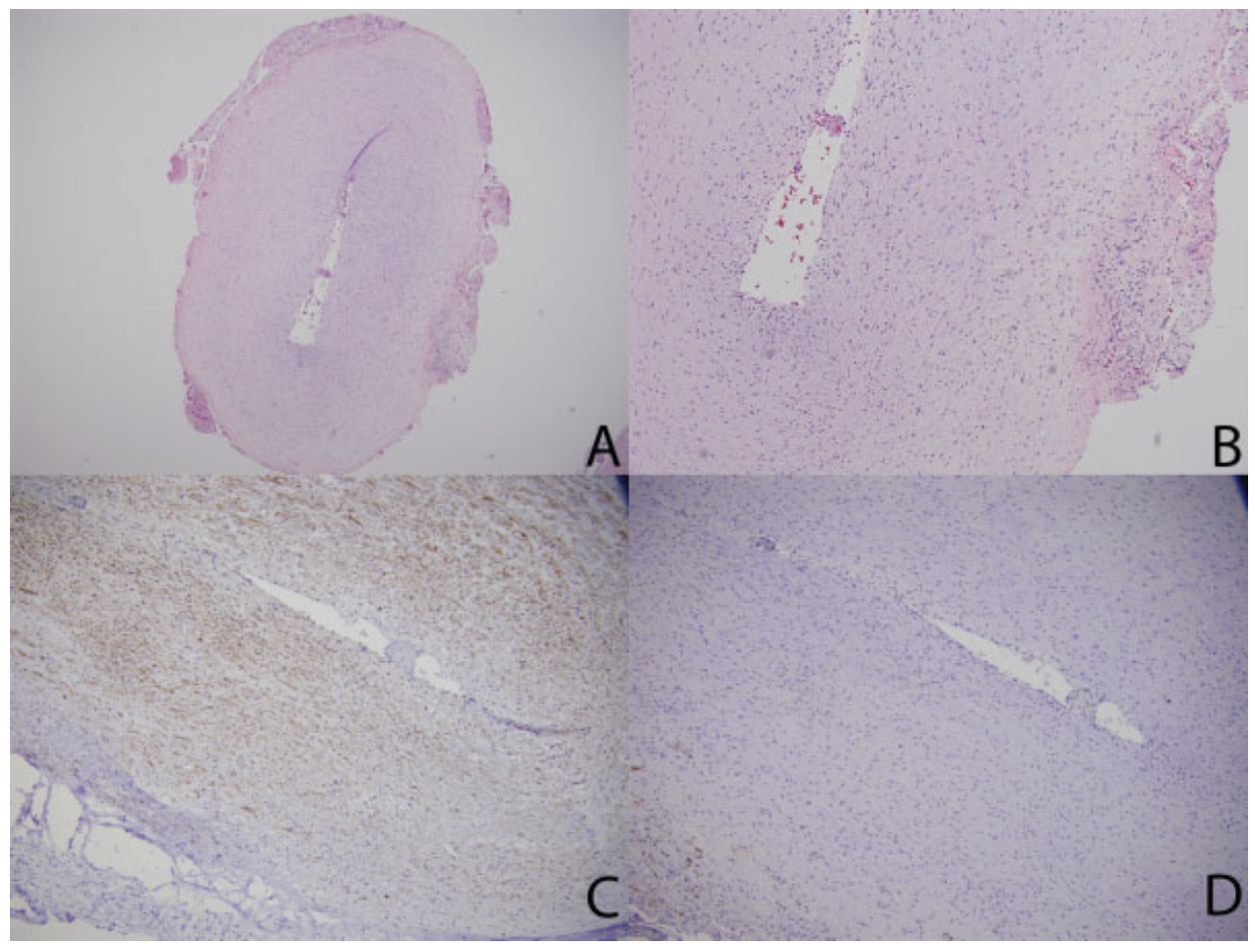

Fig. 3 (A) Hematoxylin and eosin (H\&E) stain, low power, demonstrating luminal structure of vascular mass. (B) H\&E stain, high power, demonstrating muscular wall of vascular mass consistent with artery. (C) Human muscle actin immunohistochemistry, high power, demonstrating positive staining of smooth muscle cells of the arterial muscle wall. (D) S-100 immunohistochemistry, high power, demonstrating absence of staining, thereby negating a diagnosis of schwannoma, which would stain avidly positive.

surgeons to counsel such patients and approach treatment decisions more comprehensively. Early identification and intervention via decompression of the ICA and corresponding facial nerve can lead to favorable long-term results, as demonstrated in this seminal case report.

\section{References}

1 Donaldson JA, Duckert LG, Lambert PM, Rubel EW. Anson-Donaldson: Surgical Anatomy of the Temporal Bone. 4th ed. Philadelphia, PA: Lippincott Williams and Wilkins; 1992:173-174

2 Schuknecht HF. Pathology of the Ear. Cambridge, MA: Harvard University Press; 1974:61-62 